OPEN

SUBJECT AREAS:

PLASMA PHYSICS

STATISTICAL PHYSICS

APPLIED MATHEMATICS

Received

4 November 2013

Accepted

13 February 2014

Published

7 March 2014

Correspondence and requests for materials should be addressed to A.B.M. (tony.murphy@ csiro.au)

\section{Calculation and application of combined diffusion coefficients in thermal plasmas}

Anthony B. Murphy

CSIRO Materials Science and Engineering, PO Box 218, Lindfield NSW 2070, Australia.

The combined diffusion coefficient method is widely used to treat the mixing and demixing of different plasma gases and vapours in thermal plasmas, such as welding arcs and plasma jets. It greatly simplifies the treatment of diffusion for many gas mixtures without sacrificing accuracy. Here, three subjects that are important in the implementation of the combined diffusion coefficient method are considered. First, it is shown that different expressions for the combined diffusion coefficients, arising from different definitions for the stoichiometric coefficients that assign the electrons to the two gases, are equivalent. Second, an approach is presented for calculating certain partial differential terms in the combined temperature and pressure diffusion coefficients that can cause difficulties. Finally, a method for applying the combined diffusion coefficients in computational models, which typically require diffusion to be expressed in terms of mass fraction gradients, is given.

T he combined diffusion coefficient method ${ }^{1}$ allows major simplifications in the modelling of thermal plasmas in gas mixtures, in most cases without loss of accuracy. Instead of solving conservation equations for the mass of each species (e.g., $\mathrm{N}_{2}, \mathrm{~N}_{2}{ }^{+}, \mathrm{N}, \mathrm{N}^{+}, \mathrm{N}^{2+}, \mathrm{N}^{3+}, \mathrm{Ar}, \mathrm{Ar}^{+}, \mathrm{Ar}^{2+}, \mathrm{Ar}^{3+}, \mathrm{e}^{-}$in an argon-nitrogen plasma), the species are combined into their parent gases, so only one conservation equation (e.g., for argon or nitrogen 'gas') has to be solved. The multicomponent diffusion coefficients for each pair of species (of which there are $q(q-1) / 2$ ordinary and $q-1$ thermal diffusion coefficients for a plasma containing $q$ species) are combined into just four combined diffusion coefficients, one each describing diffusion due to composition gradients, temperature gradients, pressure gradients and applied electric fields.

The method has been successfully applied to modelling a wide range of phenomena in thermal plasmas. These include demixing in welding arcs ${ }^{2,3}$, plasma torches ${ }^{4}$, arc plasma reactors ${ }^{5,6}$, and inductively-coupled rf plasmas in gas mixtures $^{7-10}$, mixing of metal vapour in welding $\operatorname{arcs}^{11-13}$, mixing of air into plasmas ${ }^{14-23}$, mixing of polymer vapours into the arc in circuit breakers ${ }^{24-29}$, and expansion of metal vapour ablated by a laser into the surrounding air ${ }^{30}$.

Values of the combined diffusion coefficients have been presented for many gas mixtures, including argonnitrogen $^{31}$, argon-oxygen ${ }^{31}$, argon-air ${ }^{32}$, argon-helium ${ }^{33,34}$, argon-hydrogen ${ }^{35}$, nitrogen-hydrogen ${ }^{36}$, sulfur hexafluoride-tetrafluoroethene ${ }^{29}$, argon-metal vapours ${ }^{11,37,38}$, and air-metal vapours ${ }^{11,39,40}$. The methods have also been extended to two-temperature plasmas ${ }^{41-43}$.

Although determination of the combined diffusion coefficients is a relatively simple extension of the calculation of the multicomponent diffusion coefficients, three points of confusion have frequently been apparent in communications with researchers performing such calculations. The first concerns the method of assigning electrons to the two gases using stoichiometric coefficients, the second the treatment of the mole fraction gradient terms in the calculation of the combined pressure and temperature diffusion coefficients, and the third the method of application of the combined diffusion coefficients in computational models, which typically require the diffusion term to be written in terms of mass fraction gradients.

The purpose of this paper is to clarify these three points. To put this in context, an introduction to the combined diffusion coefficient approach is first presented.

In the most general treatment of thermal plasmas containing more than one chemical element, the mass conservation equations for each individual species (e.g., $\mathrm{Ar}, \mathrm{Ar}^{+}, \mathrm{Ar}^{2+}, \mathrm{N}_{2}, \mathrm{~N}_{2}^{+}, \mathrm{N}, \mathrm{N}^{+}, \mathrm{N}^{2+}, \mathrm{e}^{-}$, etc., in an argonnitrogen plasma):

$$
\frac{\partial \rho Y_{i}}{\partial t}+\nabla \cdot\left(\rho \boldsymbol{v} Y_{i}\right)+\nabla \cdot \boldsymbol{J}_{i}=r_{i}
$$

have to be solved everywhere in the plasma. Here $\rho$ is the mass density, $v$ is the mass-average velocity, $Y_{i}$ is the mass fraction of species $i$, and $r_{i}$ is the net rate of production of species $i$ due to chemical reactions, vaporization, 
etc. For a gas or plasma containing q species, the diffusion mass flux of species i, $\boldsymbol{J}_{i}$, is given by ${ }^{44,45}$

$$
\boldsymbol{J}_{i} \equiv m_{i} n_{i} \boldsymbol{v}_{i}=\frac{m_{i} n^{2}}{\rho} \sum_{j=1}^{q} m_{j} D_{i j} \boldsymbol{d}_{j}-D_{i}^{T} \nabla \ln T,
$$

where $\boldsymbol{v}_{i}$ is the diffusion velocity of species i (relative to the massaverage velocity), $m_{i}$ and $n_{i}$ are respectively the mass and the number density of the species $i$, and $\boldsymbol{d}_{j}$ describes the driving forces for diffusion of species $\mathrm{j}$. The ordinary diffusion coefficients $D_{i j}$ and thermal diffusion coefficients $D_{i}^{T}$ are in this case multicomponent diffusion coefficients ${ }^{44,45}$.

For thermal plasmas, in which values of $E / n$ (electric field normalized to the gas number density) are very low (typically less than $1 \mathrm{Td}$ $=1 \times 10^{-21} \mathrm{Vm}^{2}$ ) and the species velocity distributions can be closely approximated by a Maxwellian distribution, it is standard practice to use the Chapman-Enskog approximate solution to the Boltzmann equation to obtain the diffusion coefficients. Their calculation requires values of the mole fractions and the masses of all the species present and the collision cross-sections for binary interactions between each pair of species present ${ }^{45}$. Note that the much higher values of $E / n$ present in swarms and many non-equilibrium discharges require different approaches ${ }^{46,47}$.

The driving force term $\boldsymbol{d}_{j}$ is given by ${ }^{44,45}$

$$
\boldsymbol{d}_{j}=\nabla x_{j}+\left(x_{j}-\frac{n_{j} m_{j}}{\rho}\right) \nabla \ln P-\frac{n_{j} m_{j}}{P \rho}\left(\frac{\rho}{m_{j}} \boldsymbol{F}_{j}-\sum_{l=1}^{q} n_{l} \boldsymbol{F}_{l}\right) .
$$

The three terms describe respectively diffusion due to gradients in the mole fraction $x_{j}$, the pressure $\mathrm{P}$, and the external forces $\boldsymbol{F}_{j}$ acting on species $j$. Here the only external force that is considered is that due to an applied electric field. It is interesting to note that gravity does not exert a driving force, since the third term cancels to zero.

Because diffusion velocities are defined with respect to the massaverage velocity, only $N-1$ need be calculated, and (1) has to be solved for only $N-1$ species. In plasmas, the diffusion coefficients have to be modified to take into account ambipolar diffusion ${ }^{1,45}$. This arises because electrons diffuse more rapidly than ions because of their lower mass, inducing an electric field that accelerates the ions and slows the electrons.

Clearly, solving the conservation equations (1) for each species, and calculating ordinary diffusion coefficient for each pair of species and thermal diffusion coefficients for each species, is computationally expensive. As a consequence, simplified methods are generally used; a range of methods of varying levels of simplicity and accuracy is available ${ }^{48,49}$. The combined diffusion coefficient approach has the advantage of being mathematically equivalent to the full multicomponent treatment if three conditions are satisfied: the gases are homonuclear, they do not react with each other, and the plasma is in local chemical equilibrium (LCE). Although different species will usually have different diffusion velocities, the imposition of LCE means that the local composition depends only on the local temperature, pressure and the relative concentrations of the two gases. For example, although nitrogen molecules and atoms diffuse at different rates, dissociation and recombination reactions will maintain the composition of nitrogen gas in accordance with LCE.

The diffusion mass flux of gas $\mathrm{A}$ is

$\overline{J_{A}}=\frac{n^{2}}{\rho} \overline{m_{A}} \overline{m_{B}}\left(\overline{D_{A B}^{x}} \nabla \overline{x_{B}}+\overline{D_{A B}^{P}} \nabla \ln P+\overline{D_{A B}^{E}} \boldsymbol{E}\right)-\overline{D_{A B}^{T}} \nabla \ln T$,

where $\overline{m_{A}}$ and $\overline{m_{B}}$ are the average masses of the heavy species of the respective gases

$$
\begin{aligned}
& \overline{m_{A}}=\sum_{k=2}^{p} m_{k} x_{k} / \sum_{k=2}^{p} x_{k} \\
& \overline{m_{B}}=\sum_{k=p+1}^{q} m_{k} x_{k} / \sum_{k=p+1}^{q} x_{k}
\end{aligned}
$$

and $\overline{x_{B}}$ is the sum of the mole fractions of the species of the gas $B$ :

$$
\begin{aligned}
& \overline{x_{A}}=\sum_{k=2}^{p}\left(1+Z_{k}\right) x_{k} \\
& \overline{x_{B}}=\sum_{k=p+1}^{q}\left(1+Z_{k}\right) x_{k}=1-\overline{x_{A}} .
\end{aligned}
$$

where $Z_{k}$ is the charge number of species $\mathrm{k}$, and is used to divide the electrons between the two gases.

The combined ordinary diffusion coefficient $\overline{D_{A B}^{x}}$, combined pressure diffusion coefficient $\overline{D_{A B}^{P}}$, combined electric field diffusion coefficient $\overline{D_{M G}^{E}}$ and combined temperature diffusion coefficient $\overline{D_{M G}^{T}}$ describe, respectively, diffusion due to mole fraction gradients, gradients of the total pressure, externally-applied electric fields and temperature gradients. They are calculated by writing $\overline{\boldsymbol{J}_{A}}$ and $\overline{\boldsymbol{J}_{B}}$ in terms of the total mass flux of the species of gas $A$ and gas $B$ respectively. Electrons arising from ionization of gas A species are assigned to gas $\mathrm{A}$, and likewise for gas $\mathrm{B}$, using the stoichiometric coefficients $k_{i}$ and $k_{i}^{\prime}$

$$
\begin{aligned}
& \overline{\boldsymbol{J}_{A}}=\sum_{i=1}^{p} k_{i} \boldsymbol{J}_{i}, \\
& \overline{\boldsymbol{J}_{B}}=k_{1}^{\prime} \boldsymbol{J}_{1}+\sum_{i=p+1}^{q} k_{i}^{\prime} \boldsymbol{J}_{i}=\sum_{i=1, i=p+1}^{q} k_{i}^{\prime} \boldsymbol{J}_{i},
\end{aligned}
$$

where

$$
\begin{aligned}
& k_{1}=\sum_{k=2}^{p} Z_{k} x_{k} / x_{1}, \\
& k_{1}^{\prime}=\sum_{k=p+1}^{q} Z_{k} x_{k} / x_{1}, \\
& k_{i}, k_{i}^{\prime}=1, \quad i \neq 1 .
\end{aligned}
$$

The terms $k_{i}$ and $k_{i}^{\prime}$ are used to apportion the electrons between gas A and gas $\mathrm{B}$ according to the degree of ionization of the respective gas. Using

$$
\nabla x_{j}=\left(\partial x_{j} / \partial \overline{x_{B}}\right) \nabla \overline{x_{B}}+\left(\partial x_{j} / \partial P\right) \nabla P+\left(\partial x_{j} / \partial T\right) \nabla T,
$$

which is valid since our assumption of LCE means that an equilibrium composition can be defined as a function of temperature, pressure, and the relative concentrations of the two gases, we obtain ${ }^{1,50}$ :

$$
\begin{gathered}
\overline{D_{A B}^{x}}=\frac{1}{\overline{m_{A}} \overline{m_{B}}} \sum_{i=1}^{p} k_{i} m_{i} \sum_{j=1}^{q} m_{j} D_{i j}^{a} \frac{\partial x_{i}}{\partial \overline{x_{B}}}, \\
\overline{D_{A B}^{P}}=\frac{1}{\overline{m_{A}} \overline{m_{B}}} \sum_{i=1}^{p} k_{i} m_{i} \sum_{j=1}^{q} m_{j} D_{i j}^{a}\left(x_{j}-\frac{\rho_{j}}{\rho}+P \frac{\partial x_{j}}{\partial P}\right), \\
\overline{D_{A B}^{E}}=-\frac{e}{k_{B} T} \frac{1}{\overline{m_{A}} \overline{m_{B}}} \sum_{i=1}^{p} k_{i} m_{i} \sum_{j=1}^{q} m_{j} x_{j} Z_{j} D_{i j},
\end{gathered}
$$

and

$$
\overline{D_{A B}^{T}}=\sum_{i=1}^{p} k_{i}\left(D_{i}^{T a}-\frac{n^{2}}{\rho} m_{i} \sum_{j=1}^{q} m_{j} D_{i j}^{a} T \frac{\partial x_{j}}{\partial T}\right) .
$$

We can obtain analogous expressions for $\overline{D_{B A}^{x}}, \overline{D_{B A}^{P}}, \overline{D_{B A}^{E}}$ and $\overline{D_{B A}^{T}}$ that satisfy $\overline{D_{A B}^{x}}=\overline{D_{B A}^{x}}, \overline{D_{A B}^{P}}=-\overline{D_{B A}^{P}}, \overline{D_{A B}^{E}}=-\overline{D_{B A}^{E}}$, and $\overline{D_{A B}^{T}}=-\overline{D_{B A}^{T}}$. 


\section{Results}

Stoichiometric coefficients. In previous publications $s^{1,3,32,35,48,49}$, different expressions for the combined diffusion coefficients have been given. The derivation of the coefficients was performed using number fluxes rather than mass fluxes, leading to the following expressions:

$$
\begin{gathered}
\overline{D_{A B}^{x}}=\frac{1}{\overline{m_{B}}} \sum_{i=1}^{p} s_{i} \sum_{j=1}^{q} m_{j} D_{i j}^{a} \frac{\partial x_{i}}{\partial \overline{x_{B}}}, \\
\overline{D_{A B}^{P}}=\frac{1}{\overline{m_{B}}} \sum_{i=1}^{p} s_{i} \sum_{j=1}^{q} m_{j} D_{i j}^{a}\left(x_{j}-\frac{\rho_{j}}{\rho}+P \frac{\partial x_{j}}{\partial P}\right), \\
\overline{D_{A B}^{E}}=-\frac{e}{k_{B} T} \frac{1}{\overline{m_{B}}} \sum_{i=1}^{p} s_{i} \sum_{j=1}^{q} m_{j} x_{j} Z_{j} D_{i j},
\end{gathered}
$$

and

$$
\overline{D_{A B}^{T}}=\overline{m_{A}} \sum_{i=1}^{p} s_{i}\left(\frac{D_{i}^{T a}}{m_{i}}-\frac{n^{2}}{\rho} \sum_{j=1}^{q} m_{j} D_{i j}^{a} T \frac{\partial x_{j}}{\partial T}\right)
$$

where the $s_{i}$ are stoichiometric coefficients, defined by

$$
\begin{aligned}
& s_{1}=\frac{m_{1}}{\overline{m_{A}}} \sum_{k=2}^{p} \frac{Z_{k} x_{k}}{x_{1}}, \\
& s_{i}=b_{i} \sum_{k=2}^{p} x_{k} / \sum_{k=2}^{p} b_{k} x_{k}, \quad 2 \leq i \leq p, \\
& s_{i}=b_{i} \sum_{k=p+1}^{q} x_{k} / \sum_{k=p+1}^{q} b_{k} x_{k}, p+1 \leq i \leq q
\end{aligned}
$$

where $b_{i}$ is the number of atoms in a molecule of species $i$; thus $b_{i}=2$ for a diatomic molecule or ion, and $b_{i}=1$ for an atom or monatomic ion.

In the initial publications, $s_{1}=0$ was used ${ }^{1,48}$. This is equivalent to neglecting the mass flux of the electrons $J_{1}=m_{1} n_{1} \boldsymbol{v}_{1}$, which is reasonable when calculating $\overline{D_{A B}^{x}}, \overline{D_{A B}^{P}}$ and $\overline{D_{A B}^{T}}$, since the electron mass is much smaller than the heavy species mass, and the electron diffusion velocity is of the same order of that of the heavy species due to ambipolar effects. However, ambipolar effects do not play a role in electric field diffusion, meaning that $v_{1} \gg v_{i}(i \neq 1)$, so $J_{1}$ cannot be neglected in calculating $\overline{D_{A B}^{E}}$.

The expressions for the combined diffusion coefficients given in equations (14) to (17) are in fact equivalent to the current expressions (10) to (13). We show here that the expressions given in equations (10) and (14) for $\overline{D_{A B}^{x}}$ are equal; the expressions for the other coefficients can be shown to be equal in a similar manner.

Substituting equation (18) into equation (14) gives

$\overline{D_{A B}^{x}}=\frac{m_{1}}{\overline{m_{A}} \overline{m_{B}}} \sum_{i=2}^{p} \frac{Z_{i} x_{i}}{x_{1}} \sum_{j=1}^{q} m_{j} D_{i j}^{a} \frac{\partial x_{i}}{\partial \overline{x_{B}}}+\frac{1}{\overline{m_{B}}} \sum_{i=2}^{p} b_{i} \frac{\sum_{k=2}^{p} x_{k}}{\sum_{k=2}^{p} b_{k} x_{k}} \sum_{j=1}^{q} m_{j} D_{i j}^{a} \frac{\partial x_{i}}{\partial \overline{x_{B}}}$

Since $b_{i} / b_{j}=m_{i} / m_{j}$ for species derived from the same gas, this can be written

$\overline{D_{A B}^{x}}=\frac{m_{1}}{\overline{m_{A}} \overline{m_{B}}} \sum_{i=2}^{p} \frac{Z_{i} x_{i}}{x_{1}} \sum_{j=1}^{q} m_{j} D_{i j}^{a} \frac{\partial x_{i}}{\partial \overline{x_{B}}}+\frac{1}{\overline{m_{B}}} \frac{\sum_{k=2}^{p} x_{k}}{\sum_{k=2}^{p} m_{k} x_{k}} \sum_{i=2}^{p} m_{i} \sum_{j=1}^{q} m_{j} D_{i j}^{a} \frac{\partial x_{i}}{\partial \overline{x_{B}}}$ which can be shown to be equivalent to (10) using equations (5) and (8).

Calculation of partial differentials in the expressions for combined diffusion coefficients. Equation (9) is more formally written as

$\nabla x_{j}=\left.\left(\partial x_{j} / \partial \overline{x_{B}}\right)\right|_{P, T} \nabla \overline{x_{B}}+\left.\left(\partial x_{j} / \partial P\right)\right|_{\overline{x_{B}}, T} \nabla P+\left.\left(\partial x_{j} / \partial T\right)\right|_{\overline{x_{B}, P}} \nabla T,(21)$

denoting that, for example, $\left(\partial x_{j} / \partial T\right)$, has to be calculated with $\overline{x_{B}}$ and $P$ held constant. Hence, equation (13) is written

$$
\overline{D_{A B}^{T}}=\sum_{i=1}^{p} k_{i}\left(D_{i}^{T a}-\left.\frac{n^{2}}{\rho} m_{i} \sum_{j=1}^{q} m_{j} D_{i j}^{a} T \frac{\partial x_{j}}{\partial T}\right|_{\overline{x_{B}}, P}\right),
$$

and similarly for equation (11).

For a given gas mixture, however, $\overline{x_{B}}$ is a function of temperature and pressure, due to the occurrence of dissociation and ionization reactions. In standard computer programs for the calculation of plasma composition, the relative mole fractions of the gases are set at a given temperature and pressure, typically $298 \mathrm{~K}$ and $1 \mathrm{~atm}$. Maintaining these mole fractions constant does not ensure that $\overline{x_{B}}$ stays constant with changes in temperature and pressure.

This problem can be solved by obtaining expressions for $\left.\left(\partial x_{j} / \partial T\right)\right|_{\overline{x_{B}}, P}$ and $\left.\left(\partial x_{j} / \partial P\right)\right|_{\overline{x_{B}}, T}$ in terms of the value of $\overline{x_{B}}$ at a particular temperature and pressure. These can be derived using the identity

$$
\left.\frac{\partial f}{\partial t}\right|_{s}=\left.\left.\frac{\partial f}{\partial x}\right|_{y} \frac{\partial x}{\partial t}\right|_{s}+\left.\left.\frac{\partial f}{\partial y}\right|_{x} \frac{\partial y}{\partial t}\right|_{s}
$$

where $f=f(x, y)$, with $x=x(s, t)$ and $y=y(s, t)$.

If $x=t$, so $y=y(s, x)$, this gives

$$
\left.\frac{\partial f}{\partial x}\right|_{s}=\left.\frac{\partial f}{\partial x}\right|_{y}+\left.\left.\frac{\partial f}{\partial y}\right|_{x} \frac{\partial y}{\partial x}\right|_{s}
$$

Substituting $x_{j}$ for $f, T$ for $x, \bar{x}_{B}$ for $y$, and $\overline{x_{298 \mathrm{~K}}}$ for $s$, we obtain

$\left.\left(\partial x_{j} / \partial T\right)\right|_{\overline{x_{B}}, P}=\left.\left(\partial x_{j} / \partial T\right)\right|_{\overline{x_{298 \mathrm{~K}}, P}}-\left.\left(\partial x_{j} / \partial \overline{x_{B}}\right)\right|_{P, T} \times\left.\left(\partial \overline{x_{B}} / \partial T\right)\right|_{\overline{x_{298}}, P}$

where $\overline{x_{298} \mathrm{~K}}$ is the value of $\overline{x_{B}}$ at $298 \mathrm{~K}$.

Similarly, we obtain

$\left.\left(\partial x_{j} / \partial P\right)\right|_{\overline{x_{B}, T}}=\left.\left(\partial x_{j} / \partial P\right)\right|_{\overline{x_{1 a t m}, T}}-\left.\left(\partial x_{j} / \partial \overline{x_{B}}\right)\right|_{P, T} \times\left.\left(\partial \overline{x_{B}} / \partial P\right)\right|_{\overline{x_{1 a t m}}, T}$

where $\overline{x_{1 \mathrm{~atm}}}$ is the value of $\overline{x_{B}}$ at $1 \mathrm{~atm}$.

The values of $\left(\partial x_{j} / \partial T\right)$ in equation (13) and $\left(\partial x_{j} / \partial P\right)$ in equation (11) are then calculated using equations (25) and (26), respectively.

Figure 1 shows a comparison the combined temperature diffusion coefficient calculated with and without the 'correction term', i.e., with and without the second term on the right-hand side of equation (25). The collision integrals and methods used were those given $\mathrm{in}^{31}$, as updated $\mathrm{in}^{36}$. It is clear that the 'correction term' is very significant. The corresponding term in equation (26), used in calculating the combined pressure diffusion coefficient, is in contrast, negligible, because $\overline{x_{B}}$ depends only weakly on pressure.

Application of combined diffusion coefficients in computational models. The combined diffusion coefficient approach allows us to calculate $\overline{J_{A}}$, the diffusion mass flux of gas A. The conservation equation for gas A, replacing the conservation equations (1) for each species $i$, can be written.

$$
\frac{\partial \rho \overline{Y_{A}}}{\partial t}+\nabla \cdot\left(\rho \boldsymbol{v} \overline{Y_{A}}\right)=-\nabla \cdot \overline{\boldsymbol{J}_{A}}+S_{A}
$$


where $\overline{Y_{A}}$ is the sum of mass fractions of the species making up gas $\mathrm{A}$ and $S_{A}$ is the net rate of production for gas A (for example by vaporization of a surface).

For use in standard computational algorithms, $\overline{\boldsymbol{J}_{A}}$ has to be written in terms of $\overline{Y_{A}}$. We can modify Eq. (4) to read

$\overline{J_{A}}=-\rho \frac{\overline{m_{A}} \overline{m_{B}}}{\bar{M}^{2}}\left(\overline{D_{A B}^{x}} \nabla \overline{x_{A}}-\overline{D_{A B}^{P}} \nabla \ln P-\overline{D_{A B}^{E}} \boldsymbol{E}\right)-\overline{D_{A B}^{T}} \nabla \ln T$

noting that $\nabla \overline{x_{A}}=-\nabla \overline{x_{B}}$ and $n^{2} / \rho=\rho / \bar{M}^{2}$, where $\bar{M}$ is the average mass of all species in the gas mixture

$$
M=\sum_{k=1}^{q} m_{k} x_{k}
$$

Since $\overline{Y_{A}}=\left(\overline{M_{A}} / \bar{M}\right) \overline{x_{A}}$, where $\overline{M_{A}}$ is the average mass of all species of gas A:

$$
\overline{M_{A}}=\sum_{k=2}^{p}\left(m_{k} x_{k}+m_{1} Z_{k} x_{k}\right) / \sum_{k=2}^{p}\left(1+Z_{k}\right) x_{k},
$$

we have

$$
\nabla \overline{x_{A}}=\frac{\bar{M}}{\overline{M_{A}}} \nabla \overline{Y_{A}}+\overline{Y_{A}} \nabla\left(\frac{\bar{M}}{\overline{M_{A}}}\right)
$$

Therefore,

$$
\begin{aligned}
\overline{J_{A}} & =-\rho \frac{\overline{m_{A}} \overline{m_{B}}}{\overline{M_{A}} \bar{M}} \overline{D_{A B}^{x}} \nabla \overline{Y_{A}}-\rho \frac{\overline{m_{A}} \overline{m_{B}}}{\bar{M}^{2}} \overline{D_{A B}^{x} \overline{Y_{A}}} \nabla\left(\frac{\bar{M}}{\overline{M_{A}}}\right) \\
& +\rho \frac{\overline{m_{A}} \overline{m_{B}}}{\bar{M}^{2}} \overline{D_{A B}^{P}} \ln P+\rho \frac{\overline{m_{A}} \overline{m_{B}} \overline{\bar{D}^{2}} \overline{\bar{M}^{2}} \boldsymbol{E}-\overline{D_{A B}^{T}} \nabla \ln T}{}
\end{aligned}
$$

Substituting this into equation (27) gives

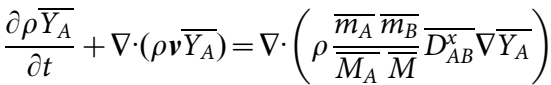

$$
\begin{aligned}
& +\nabla \cdot\left[\rho \frac{\overline{m_{A}} \overline{m_{B}}}{\bar{M}^{2}} \overline{D_{A B}^{x}} \overline{Y_{A}} \nabla\left(\frac{\bar{M}}{\overline{M_{A}}}\right)\right]
\end{aligned}
$$

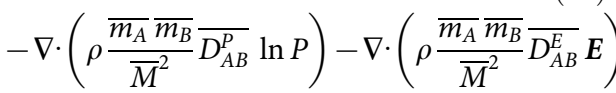

$$
\begin{aligned}
& +\nabla \cdot\left(\overline{D_{A B}^{T}} \nabla \ln T\right)+S_{A}
\end{aligned}
$$

The first term on the right-hand side is the diffusion term, while the second, third, fourth and fifth terms on the right-hand side are not in $\nabla \overline{Y_{A}}$, and therefore have to be treated as source terms.

\section{Discussion}

The following points related to the combined diffusion coefficients have been clarified.

1. Different expressions for the combined diffusion coefficients, arising from different definitions for the stoichiometric coefficients that assign the electrons to the two gases, have been shown to be equivalent;

2. An approach to dealing with the partial differential terms for which the mole fractions of the gases are held constant, which have to be calculated in determining the combined temperature and pressure diffusion coefficients, has been presented;

3. A method for the application of the combined diffusion coefficients in computational models that, as is usually the case, require diffusion to be expressed in terms of mass fraction gradients, has been described.

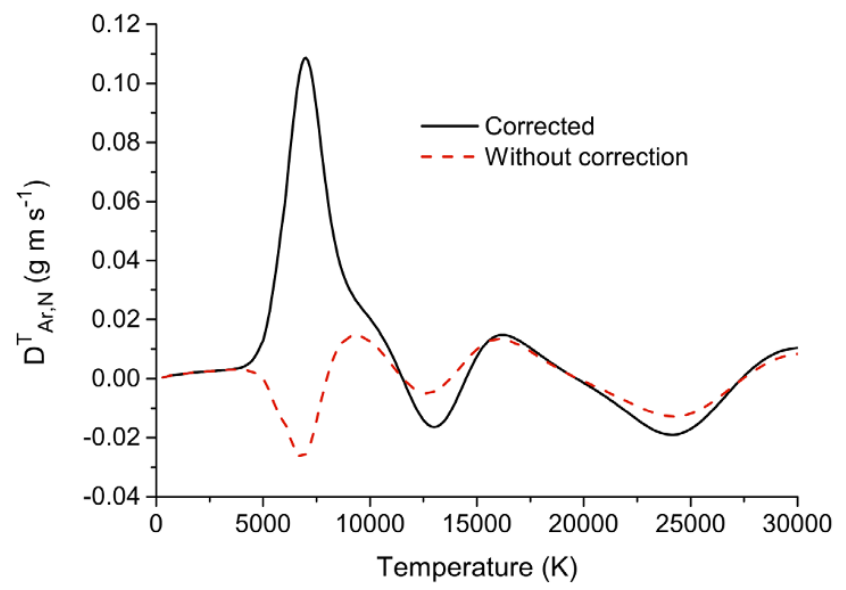

Figure $1 \mid$ Combined temperature diffusion coefficient for a mixture of equal parts argon and nitrogen by mole fraction at $1 \mathrm{~atm}$. The 'without correction' curve was calculated including only the first term on the right-hand side of equation (25), while the 'corrected' curve was calculated using the full expression.

The first point is an important consideration in the consistent definition of the combined diffusion coefficients, and the correct choice of stoichiometric coefficients becomes critical when diffusion driven by external electric fields is taken into account.

The second point has to be understood and implemented to correctly calculate the combined temperature and pressure diffusion coefficients. In particular, very large errors are introduced in the calculation of the combined temperature diffusion coefficient if this point is neglected.

Finally, the third point provides an approach to implement the combined diffusion coefficients into standard computational models of thermal plasmas.

1. Murphy, A. B. Diffusion in equilibrium mixtures of ionized gases. Phys. Rev. E 48, 3594-3603 (1993).

2. Murphy, A. B. Demixing in free-burning arcs. Phys. Rev. E 55, 7473-7494 (1997)

3. Murphy, A. B. Cataphoresis in electric arcs. J. Phys. D: Appl. Phys. 31, 3383-3390 (1998).

4. Ghorui, S., Vysohlid, M., Heberlein, J. V. R. \& Pfender, E. Probing instabilities in arc plasma devices using binary gas mixtures. Phys. Rev. E 76, 016404 (2007).

5. Fudolig, A. M., Nogami, H. \& Yagi, J. Modeling of the flow, temperature and concentration fields in an arc plasma reactor with argon-nitrogen atmosphere. ISIJ Int. 36, 1222-1228 (1996).

6. Fudolig, A. M., Nogami, H., Yagi, J., Mimura, K. \& Isshiki, M. Prediction of surface temperature on metal beads subjected to argon-hydrogen transferred arc plasma impingement. ISIJ Int. 37, 623-629 (1997)

7. Colombo, V., Ghedini, E. \& Sanibondi, P. Three-dimensional investigation of particle treatment in an RF thermal plasma with reaction chamber. Plasma Sources Sci. Technol. 19, 065024 (2010).

8. Colombo, V. et al. Fluid-dynamic characterization of a radio-frequency induction thermal plasma system for nanoparticle synthesis. Plasma Sources Sci. Technol. 21, 045010 (2012).

9. Colombo, V., Ghedini, E., Gherardi, M. \& Sanibondi, P. Modelling for the optimization of the reaction chamber in silicon nanoparticle synthesis by a radiofrequency induction thermal plasma. Plasma Sources Sci. Technol. 21, 055007 (2012).

10. Chen, X., Sugasawa, M. \& Kikukawa, N. Modelling of the heat transfer and fluid flow in a radio-frequency plasma torch with argon-hydrogen as the working gas. J. Phys. D: Appl. Phys. 31, 1187-1196 (1998).

11. Murphy, A. B. The effects of metal vapour in arc welding. J. Phys. D: Appl. Phys. 43, 434001 (2010).

12. Schnick, M. et al. Modelling of gas-metal arc welding taking into account metal vapour. J. Phys. D: Appl. Phys. 43, 434008 (2010).

13. Murphy, A. B. Influence of metal vapour on arc temperatures in gas-metal arc welding: convection versus radiation. J. Phys. D: Appl. Phys. 46, 224004 (2013).

14. Li, H. P. \& Chen, X. Three-dimensional modeling of the turbulent plasma jet impinging upon a flat plate and with transverse particle and carrier-gas injection. Plasma Chem. Plasma Process. 22, 27-58 (2002). 
15. Cheng, K. \& Chen, X. Prediction of the entrainment of ambient air into a turbulent argon plasma jet using a turbulence-enhanced combined-diffusion-coefficient method. Int. J. Heat Mass. Transf. 47, 5139-5148 (2004).

16. Cheng, K. \& Chen, X. Numerical prediction of the noise emission from a turbulent argon thermal-plasma jet issuing into ambient air. Plasma Chem. Plasma Process. 25, 677-698 (2005)

17. Xu, D. Y. \& Chen, X. Effects of surrounding gas on the long laminar argon plasma jet characteristics. Int. Comm. Heat Mass Transf. 32, 939-946 (2005).

18. Cheng, K., Chen, X. \& Pan, W. X. Comparison of laminar and turbulent thermal plasma jet characteristics - A modeling study. Plasma Chem. Plasma Process. 26, 211-235 (2006).

19. Wang, H. X., Cheng, K., Chen, X. \& Pan, W. X. Three-dimensional modeling of heat transfer and fluid flow in laminar-plasma material re-melting processing. Int. J. Heat Mass. Transf. 49, 2254-2264 (2006).

20. Wang, H.-X., Chen, X., Cheng, K. \& Pan, W. Modeling study on the characteristics of laminar and turbulent argon plasma jets impinging normally upon a flat plate in ambient air. Int. J. Heat Mass. Transf. 50, 734-745 (2007).

21. Wang, H.-X., Chen, X. \& Pan, W. Modeling study on the entrainment of ambient air into subsonic laminar and turbulent argon plasma jets. Plasma Chem. Plasma Process. 27, 141-162 (2007).

22. Wang, H.-X., Chen, X. \& Pan, W. Effects of the length of a cylindrical solid shield on the entrainment of ambient air into turbulent and laminar impinging argon plasma jets. Plasma Chem. Plasma Process. 28, 85-105 (2008).

23. Kai, C. \& Xi, C. Effects of natural convection on the characteristics of long laminar argon plasma jets issuing upwards or downwards into ambient air - a numerical study. J. Phys. D: Appl. Phys. 37, 2385-2391 (2004).

24. Ma, Q. et al. Simulation and experimental study of arc motion in a low-voltage circuit breaker considering wall ablation. IEICE Trans. Electron. E91-C, 1240-1248 (2008).

25. Rong, M., Ma, Q., Wu, Y., Xu, T. \& Murphy, A. B. The influence of electrode erosion on the air arc in a low-voltage circuit breaker. J. Appl. Phys. 106, 023308 (2009).

26. Ma, Q., Rong, M., Murphy, A. B., Wu, Y. \& Xu, T. Simulation study of the influence of wall ablation on arc behavior in a low-voltage circuit breaker. IEEE Trans. Plasma Sci. 37, 261-269 (2009).

27. Yang, F. et al. Numerical analysis of the influence of splitter-plate erosion on an air arc in the quenching chamber of a low-voltage circuit breaker. J. Phys. D: Appl. Phys. 43, 434011 (2010).

28. Yang, F. et al. Simulation of arc splitting process considering splitter-plate erosion. IEEE Trans. Plasma Sci. 39, 2862-2863 (2011).

29. Zhang, J. L., Yan, J. D., Murphy, A. B., Hall, W. \& Fang, M. T. C. Computational investigation of arc behaviour in an auto-expansion circuit breaker contaminated by ablated nozzle vapour. IEEE Trans. Plasma Sci. 30, 706-719 (2002).

30. Gomes, A., Aubreton, A., Gonzalez, J. J. \& Vacquie, S. Experimental and theoretical study of the expansion of a metallic vapour plasma produced by laser. J. Phys. D: Appl. Phys. 37, 689-696 (2004).

31. Murphy, A. B. \& Arundell, C. J. Transport coefficients of argon, nitrogen, oxygen, argon-nitrogen and argon-oxygen plasmas. Plasma Chem. Plasma Process. 14, 451-490 (1994).

32. Murphy, A. B. Transport coefficients of air, argon-air, nitrogen-air, and oxygenair plasmas. Plasma Chem. Plasma Process. 15, 279-307 (1995).

33. Aubreton, J., Elchinger, M. F., Rat, V., Fauchais, P. \& Murphy, A. B. Twotemperature combined diffusion coefficients in argon-helium thermal plasmas. High Temp. Mater. Process. 7, 107-113 (2003).
34. Murphy, A. B. Transport coefficients of helium and argon-helium plasmas. IEEE Trans. Plasma Sci. 25, 809-814 (1997).

35. Murphy, A. B. Transport coefficients of hydrogen and argon-hydrogen plasmas. Plasma Chem. Plasma Process. 20, 279-297 (2000).

36. Murphy, A. B. Transport coefficients of plasmas in mixtures of nitrogen and hydrogen. Chem. Phys. 398, 64-72 (2011).

37. Cressault, Y., Murphy, A. B., Teulet, P., Gleizes, A. \& Schnick, M. Thermal plasma properties for $\mathrm{Ar}-\mathrm{Cu}, \mathrm{Ar}-\mathrm{Fe}$ and $\mathrm{Ar}-\mathrm{Al}$ mixtures used in welding plasmas processes: Part II. Transport coefficients. J. Phys. D: Appl. Phys. 46, 415207 (2013).

38. Aubreton, A. \& Elchinger, M. F. Transport properties in non-equilibrium argon, copper and argon-copper thermal plasmas. J. Phys. D: Appl. Phys. 36, 1798-1805 (2003).

39. Cressault, Y. \& Gleizes, A. Calculation of diffusion coefficients in air-metal thermal plasmas. J. Phys. D: Appl. Phys. 43, 434006 (2010).

40. Cressault, Y., Gleizes, A. \& Riquel, G. Properties of air-aluminum thermal plasmas. J. Phys. D: Appl. Phys. 45, 265202 (2012).

41. Rat, V., Aubreton, J., Elchinger, M. F., Fauchais, P. \& Murphy, A. B. Diffusion in two-temperature thermal plasmas. Phys. Rev. E 66, 056407 (2002).

42. Zhang, Z.-N., Li, H.-P., Murphy, A. B. \& Xia, W.-D. A numerical model of nonequilibrium thermal plasmas. I. Transport properties. Phys. Plasmas 20, 033508 (2013).

43. Rat, V., Aubreton, J., Elchinger, M. F. \& Fauchais, P. Calculation of combined diffusion coefficients from the simplified theory of transport properties. Plasma Chem. Plasma Process. 21, 355-369 (2001).

44. Hirschfelder, J. O., Curtiss, C. F. \& Bird, R. B. Molecular Theory of Gases and Liquids (Wiley, 1954).

45. Devoto, R. S. Transport properties of ionized monatomic gases. Phys. Fluids $\mathbf{9}$, 1230-1240 (1966).

46. Robson, R. E. Introductory Transport Theory for Charged Particles in Gases. (World Scientific, 2006).

47. Kumar, K., Skullerud, H. R. \& Robson, R. E. Kinetic theory of charged particle swarms in neutral gases. Aust. J. Phys. 33, 343-448 (1980).

48. Murphy, A. B. A comparison of treatments of diffusion in thermal plasmas. J. Phys. D: Appl. Phys. 29, 1922-1932 (1996).

49. Murphy, A. B. Treatments of diffusion in thermal plasmas. High Temp. Mater. Process. 4, 1-20 (2000).

50. Murphy, A. B. Thermal plasmas in gas mixtures. J. Phys. D: Appl. Phys. 34 R151-R173 (2001)

\section{Author contributions}

A.B.M. performed all the research presented, prepared the figure, and wrote the manuscript.

\section{Additional information}

Competing financial interests: The authors declare no competing financial interests.

How to cite this article: Murphy, A.B. Calculation and application of combined diffusion coefficients in thermal plasmas. Sci. Rep. 4, 4304; DOI:10.1038/srep04304 (2014).

his work is licensed under a Creative Commons Attribution-

NonCommercial-NoDerivs 3.0 Unported license. To view a copy of this license, visit http://creativecommons.org/licenses/by-nc-nd/3.0 